\title{
Transcriptome sequencing of Verticillium dahliae from a cotton farm reveals positive correlation between virulence and tolerance of sugar-induced hyperosmosis
}

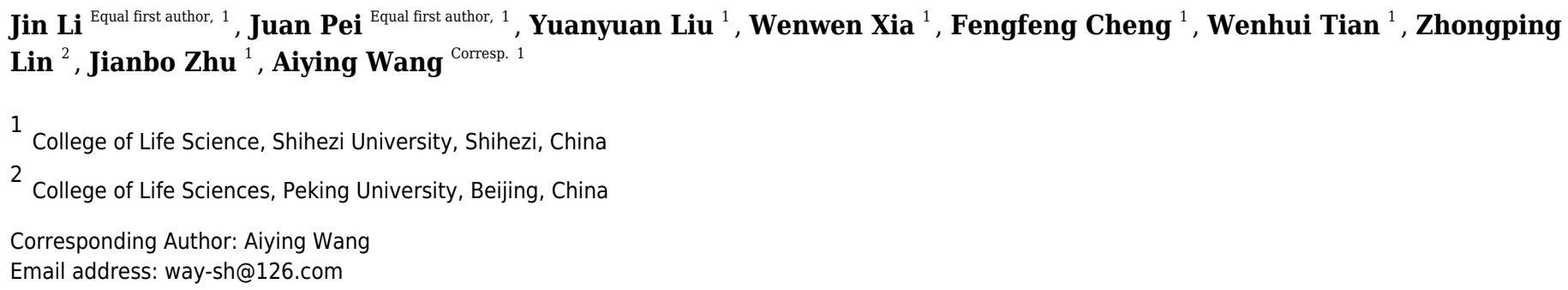

Verticillium dahliae causes disease symptoms in its host plants; however, due to its rapid variability, $V$. dahliae is difficult to control. To analyze the reason for this pathogenic differentiation, $22 \mathrm{~V}$. dahliae strains with different virulence were isolated from a cotton farm. The genetic diversity of cotton varieties make cotton cultivars have different Verticillium wilt resistance, so the Xinluzao 7 (susceptible to V. dahliae), Zhongmian 35 (tolerant), and Xinluzao 33 (resistant) were used to investigate the pathogenicity of the strains in a green house. Vegetative compatibility groups (VCGs) assays, Internal Transcribed Spacer (ITS) PCR, and pathogenicity analysis showed that SHZ-4, SHZ-5, and SHZ-9 had close kinship and significantly different pathogenicity. Transcriptome sequencing of the three strains identified 19 of 146 unigenes in SHZ-4_vs_SHZ-5, SHZ-5_vs_SHZ-9, and SHZ-4_vs_SHZ-9. In these unigenes, three proteinase and four polysaccharide degrading hydrolases were found to be associated with the pathogenicity. However, due to a number of differentially expressed genes in the transport, these unigenes not only played a role in nutrition absorption but might also contribute to the resistance of sugar-induced hyperosmosis. Moreover, the tolerance ability was positively related to the pathogenicity of $V$. dahliae. This resistance to sugar-induced hyperosmosis might help $V$. dahliae to access the nutrition of the host. The pathogenicity of $V$. dahliae correlated with the resistance of sugar-induced-hyperosmosis, which provides clues for the cultivation of $V$. dahliae resistant varieties. 
1 Transcriptome sequencing of Verticillium dahliae from a cotton farm reveals positive correlation between virulence and tolerance of sugar-induced hyperosmosis

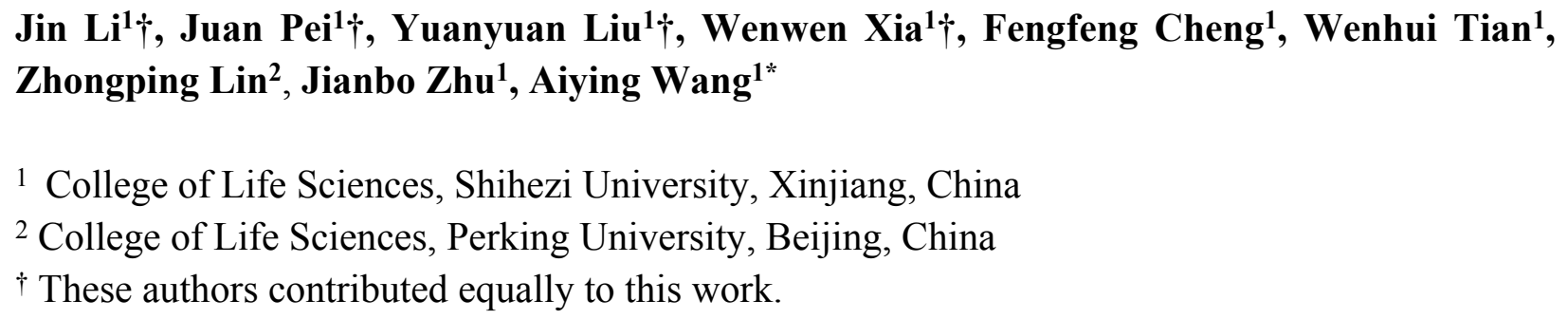

\section{Abstract}

Verticillium dahliae causes disease symptoms in its host plants; however, due to its rapid variability, $V$. dahliae is difficult to control. To analyze the reason for this pathogenic differentiation, $22 \mathrm{~V}$. dahliae strains with different virulence were isolated from a cotton farm. The genetic diversity of cotton varieties make cotton cultivars have different Verticillium wilt resistance, so the Xinluzao 7 (susceptible to $V$. dahliae), Zhongmian 35 (tolerant), and Xinluzao 33 (resistant) were used to investigate the pathogenicity of the strains in a green house. Vegetative compatibility groups (VCGs) assays, Internal Transcribed Spacer (ITS) PCR, and pathogenicity analysis showed that SHZ-4, SHZ-5, and SHZ-9 had close kinship and significantly different pathogenicity. Transcriptome sequencing of the three strains identified 19 of 146 unigenes in SHZ-4_vs_ SHZ-5, SHZ-5_vs_SHZ-9, and SHZ-4_vs_ SHZ-9. In these unigenes, three proteinase and four polysaccharide degrading hydrolases were found to be associated with the pathogenicity. However, due to a number of differentially expressed genes in the transport, these unigenes not only played a role in nutrition absorption but might also contribute to the resistance of sugar-induced hyperosmosis. Moreover, the tolerance ability was positively related to the pathogenicity of $V$. dahliae. This resistance to sugar-induced hyperosmosis might help $V$. dahliae to access the nutrition of the host. The pathogenicity of $V$. dahliae correlated with the resistance of sugar-induced-hyperosmosis, which provides clues for the cultivation of $V$. dahliae resistant varieties.

Keywords: Pathogenicity differentiation; Verticillium dahliae; Transcriptome; Transport; Hyperosmosis; Host-cell-wall degradation

\section{Introduction}


40

To stand and face various biotic and abiotic stresses, plants have evolved a wide spectrum of mechanisms to defend themselves against these stresses, especially pathogen infection. The mechanisms in response to pathogen attack are divided into two groups, pre-existing and induced. The pre-existing mechanisms mainly involve physical and chemical barriers for protecting plants from pathogen infestation at the first line, including the plant cuticle, cell wall, and antimicrobial compounds (Pieterse et al., 2009; Wang et al., 2019). Salicylic acid (SA) crosstalking with auxin, ethylene, and jasmonates (JA) is crucial for the inducible response of plants to pathogens infection including systemic acquired resistance and induced systemic resistance (Pieterse et al., 1998; Lopez et al., 2008; Vlot et al., 2009). In the plants response to Verticillium dahliae infection, many genes that are mostly involved in lignin biosynthesis and phenylpropanoid metabolic pathway are induced (Li et al., 2019b; Yang et al., 2019; Zhang et al., 2019c). The increasing of these gene expressions could confer resistance to the Verticillium wilt (VW) in cotton. For cotton varieties, the Gossypium barbadense possess innate resistance to VW and premium fiber quality, but low-fiber productivity upon infection (Sun et al., 2013). Thus, the development of new cotton cultivars possessing VW resistance by means of traditional breeding and transgenic strategies based on the pathogenic mechanism of $V$. dahliae are the most practical and cost effective method to manage cotton VW.

Verticillium dahliae is a fungal plant pathogen with wide global distribution and the ability to infect a number of important cash crops such as cotton, tomato, sunflower, and sugar beet, causing significant yield reduction (Fradin and Thomma, 2006; Klosterman et al.,2009). In response to an adverse environment, it produces a dormant structure (the microsclerotia) that can survive many years in cultivated soil and its germination is induced by exudates of plant roots (Cai et al., 2009; Klosterman et al., 2011). Upon induction, hyphae of $V$. dahliae cover the plant root surface and penetrate the root epidermal cells of susceptible plants (Klimes and Dobinson, 2006). Thereafter, the hyphae proliferate in the vascular system, which causes severe wilting of plants (Huisman, 1982; Sanei et al., 2005). Given the economic losses of cash crop production as a result of $V$. dahliae infection, understanding the mechanism of its pathogenicity is an urgent requirement.

$V$. dahliae causes apparent disease symptoms, which often include wilt and chlorosis, plant stunting, and vascular discoloration. Moreover, pathogenicity varied between hosts (Fradin and Thomma, 2006). Due to its broad host range, $V$. dahliae was assumed to have large genetic 
71 diversity. Thereafter, an increasing number of vegetative compatibility assays and molecular 72 research illustrated that $V$. dahliae indeed had significant genetic diversity (Bao et al., 1998; 73 Korolev et al., 2001; Rowe, 1995; Baroudy et al., 2019). In the study by Göre (2007), 101 74 filtered isolates were assigned to four vegetative compatibility groups (VCGs), the 75 pathogenicities of which ranged from weak to strong. Korolev et al. (2001) identified 62 isolates 76 from Spain and 49 from Israel, which were classified into four VCG groups via VCG assays. 77 Among each group, the virulence of different isolates ranged from weak to strong. Previously, 78 strains from cotton had been classified into two groups based on symptoms (defoliation and non79 defoliation) (Schnathorst and Mathre, 1966). Daayf et al. (1995) further classied the V. dahliae into four groups via their vegetative compatibility and pathogenicity on cotton. Recently, Dung et al., (2019) using genotyping by sequencing, PCR assays for mating-type and pathogenic race, vegetative compatibility group (VCG) tests, and aggressiveness assays identified the genetic diversity of $V$. dahliae. The diversity at the population level was attributed to the ability for rapid genetic variation, which called for the development of varieties that are resistant to $V$. dahliae infection due to the emergence of newly resistant strains.

Today, as a result of the development of omics technology, many of the genes that are responsible for the pathogenicity were identified. Chu et al. identified 106 secreted proteins from $V$. dahliae under nutrition stress (Chu et al., 2015). These proteins are involved in cell wall degradation, the scavenging of and stress response to reactive oxygen species (ROS), lipid effectors, protein metabolism, carbohydrate metabolism, electron-proton transport, and energy metabolism. Chen et al. (2016) investigated the exoproteome via cotton-containing medium induction, where the identified 271 secreted proteins were enriched in carbohydrate hydrolyses and carbohydrate-active (CAZymes) involved in pectin and cellulose degradation pathways. These proteins play central roles in the pathogenicity. Hydrolases involved in plant cell wall degradation were regarded as criteria proteins for the production of disease symptoms and pathogenicity (King et al., 2011; Glass et al., 2013; Kubicek et al., 2014; Zhang et al., 2019a). Knock down of sucrose non-fermenting 1 ( $V d S N F 1)$ reduced expression of a number of PCWDEs and strongly impaired pathogenicity (Tzima et al., 2011). In addition, many genes were identified that play a role in pathogenicity, such as VMK1,VdPKAC1, VdSge1, and VdMFS that mainly function in adhesion and penetration (Luo et al., 2014). However, these findings cannot explain the virulence differentiation of the $V$. dahliae sufficiently. The DNA 
102 rearrangement and lineage-specific (LS) genomic regions are related to virulence and niche 103 adaptation, and the major generators are transposable elements (TEs) (de Jonge et al., 2013).

104 This promotes the rapid development of genes that are relevant to the host adaptation and 105 virulence (Klosterman et al., 2011; de Jonge et al., 2013; Amyotte et al., 2012). Chen et al. (2017) 106 confirmed that horizontal gene transfer from Fusarium to Vd991 significantly improved the 107 ability for host adaptation.

108 Although studies on the pathogenicity and variation of $V$. dahliae have achieved 109 considerable progress, the variation mechanism and key genes responsible for the different 110 virulence of strains remain unclear. The following key problems remain: 1. The genetic 111 background of $V$. dahliae species that were compared was not consistent - the majority of studies 112 investigated $V$. dahliae collected from different regions; 2. $V$. dahliae possesses the ability for 113 rapid variation. Therefore, the genes that play the main role in the pathogenicity differentiation 114 remain unknown. The completion of $V$. dahliae genome sequencing enables the accurate study of 115 the variation mechanism (Chen et al., 2017). To investigate the key variation gene between $V$. 116 dahliae strains with high close genetic relationship and different pathogenicities, strains were 117 collected from a cotton field of the Shihezi region, with the aim to reduce differences in the 118 genetic background. VCGs and specific marker PCR were used to assure kinship. Virulence 119 testing of all strains on three cotton varieties was used to analyze the pathogenicity differences of 120 isolates. Through genetic relationship and pathogenicity differentiation, three isolates were 121 selected for transcriptome sequencing with the aim to seek the key gene responsible for 122 pathogenicity differentiation.

123

124 Materials \& Methods

\section{Molecular identification of $V$. dahliae}

126

Field experiments was approved by Xinjiang Han, a personal farmer. To collect the $V$. 127 dahliae strains with close relationship, infected cotton plants were collected from a field that the 128 Verticillium wilt was serious in recent decades in the Shihezi region, Xinjiang province (E 86.04, $129 \mathrm{~N} \mathrm{44.30)}$. The stems were separated into 0.5-1 cm slices, after which they were disinfected via $130 \mathrm{HgCl}_{2}$ for 10-15 min. PDA medium was used to culture $V$. dahliae. The CTAB method was used 131 to extract the DNA of $V$. dahliae. The ITS sequence was obtained via ITS1/ITS4 universal 
132 primer PCR. PCR was conducted using the following protocol: f $95{ }^{\circ} \mathrm{C}$ for $10 \mathrm{~min}$; 30 cycles of $13395{ }^{\circ} \mathrm{C}$ for $45 \mathrm{~s}, 56^{\circ} \mathrm{C}$ for $30 \mathrm{~s}, 72{ }^{\circ} \mathrm{C}$ for $30 \mathrm{~s}$, and $72{ }^{\circ} \mathrm{C}$ for $10 \mathrm{~min}$. All ITS sequences were 134 cloned into pMD19-T vector and sequenced. The Neighbor-joining model was used to construct 135 the phylogenetic tree by ClustalW of the software Mega 5.0, using the default cutoff.

136 Vegetative Compatibility Groups (VCGs) Assay

137 All $V$. dahliae strains were used to obtain the Nit mutant strains. The process was conducted 138 according to the method described in Joaquim et al. (1990). All obtained Nit mutant strains were 139 used to assess the vegetative compatibility relationships of $V$. dahliae strains. The experimental 140 procedure followed the method described in Joaquim et al. (1991). All the experiments were 141 repeated three times.

\section{Pathogenicity assay on cotton plants}

143 Xinluzao 7 (susceptible to the pathogen), Zhongmian 35 (tolerant), and Xinluzao 33 144 (resistant) were used to investigate the pathogenicity of $V$. dahliae in a green house of key 145 laboratory of Shihezi university. The conidial concentration was estimated via hemocytometer 146 and adjusted to $1 * 10^{7} \mathrm{CFU} / \mathrm{ml}$. The experimental procedure and calculation of disease index 147 were conducted according to Zhu et al. (2013). All experiments were repeated three times. 148 Variance analysis was conducted using SPSS v17.0 (SPSS Inc.; Chicago, IL, USA).

\section{Transcriptome sequencing of $\boldsymbol{V}$. dahliae}

150 The SHZ-4, SHZ-5, and SHZ-9 strains was cultured in the Czapek liquid medium for 14 151 days at the conditions of $25^{\circ} \mathrm{C}$, dark, and $180 \mathrm{rpm} / \mathrm{min}$. The spore suspension was abtained through filtering the hyphae off. The spore suspension was then centrifuged to collect the concentrated spores. The total RNA were extracted using the Fungal RNA miniprep kit (Biomiga, San Diego, USA) according to the manufacturer's instructions. Total RNA was sent to the Sangon Biotech Cooperation (Shanghai, China) for RNA examination and sequencing. The sequencing platform was a HiSeq-2500-Pe-125 of Illumina. The total raw data was processed by the FASTQC software with default parameters. Cutadapt software used $\mathrm{O}=10$, min len $=35$, a = adaptor sequence. Prinseq software used the following parameter definitions: trim_qual_left $=$ 20 , trim_qual_right $=20$, trim_qual_window $=10$, trim_qual_step $=1$, and the Blast + with an e- 
161 assembled by the Trinity software (http://trinityrnaseq.github.io/) with min_contig_length set to 162 200. The longest transcript was considered as a unigene. All unigenes were successfully 163 annotated with a similarity $>30 \%$ and an e-value $=1 \mathrm{e}-5$. Blastn (NCBI blast2.2.28+) was used 164 against the NT database. For the unigenes BlastX (NCBI blast2.2.28+) was used against NR, 165 SwissProt, and TrEMBL databases. For CDD, COG/KOG, and PFAM annotations, the rpsblast 166 software (NCBI blast2.2.28+) was used. GO annotation was obtained according to the annotated

167 168 169

170

171

172

173

174

175 176

177

178

179

180

181

182

183

184

185

186

187

188

189

190 result of both SwissProt and TrEMBL. The KEGG Automatic Annotation Server of KAAS was used for KEGG annotation. The RSEM/bowtie program (version 1.2.8; Madison, WI, USA) with a cutoff defined at $\mathrm{v}=2$ software was used for the mapping and the RSeQC (http://rseqc.sourceforge.net/) was used for the statistic of the mapping with default settings. The expression level of unigenes (Fragment Per Kilo bases per Million mapped Reads, FPKM) was calculated by the RSEM/bowtie program with default settings. Since the transcriptome sequencing had no biological repetition, the analysis of differential unigene expression was conducted in reference to the Audic method (Audic and Claverie,1997). The DESeq and edgeR programs were used to identify the differential expression of unigenes and the cutoff was defined at $\mathrm{p} \leq 0.01, \log \mathrm{FC} \geq 2$, and FDR $<0.001$. The figure was merged by the Photoshop_CS3_SC_V1.3 (Adobe System Incorporation; San Jose, CA, USA).

\section{Validation of RNA-Seq Data by qRT-PCR}

Six unigenes being selected randomly from the data that were identified in all SHZ-4_vs SHZ-5, SHZ-5_vs_SHZ-9, and SHZ-4_vs_SHZ-9 DEG data were used to confirm the expression patterns of the Illumina RNA-Seq results by quantitative real-time PCR (qRT-PCR). The ROCHE LightCycler® 480 system (Salt Lake City, UT, USA) was used to determine the expression level of the selected genes and the SYBR Green Real-Time PCR Master Mix (KAPA Biosystems, Wilmington, MA, USA) was utilized in $10 \mu \mathrm{L}$ reactions. The reaction system consisted of $2 \mathrm{ng}$ template, $0.8 \mu \mathrm{L}$ primers, and $5 \mu \mathrm{L}$ master mixes. The PCR reactions were performed in a thermocycler using the following conditions: $5 \mathrm{~min}$ at $95^{\circ} \mathrm{C}, 45$ cycles of $10 \mathrm{~s}$ at $95{ }^{\circ} \mathrm{C}, 15 \mathrm{~s}$ at $60{ }^{\circ} \mathrm{C}$, and $25 \mathrm{~s}$ at $72{ }^{\circ} \mathrm{C}$. Primers were designed with Primer Premier software (Primer Premier v5.0; Premier Biosoft International, Palo Alto, CA, USA). The $\beta$-tubulin gene was used as reference. The experiments were repeated three times. To assess the correlation between transcriptome sequencing and quantitative Real-Time PCR (qRT-PCR), Pearson's 
191 coefficient was calculated using OriginPro 8.6 (OriginLab; Northampton, MA, USA). The figure 192 was merged by the Photoshop_CS3_SC_V1.3 (Adobe System Incorporation; San Jose, CA, 193 USA).

194 195

\section{Results}

196

197

198

199

200

201

202

203

204

205

206

207

208

209

210

211

212

213

214

215

216

217

218

\section{Molecular identification and Vegetative Compatibility Groups (VCGs) of V. dahliae}

The ITS sequences of the isolates were obtained via universal primer ITS PCR. Molecular phylogenetic tree analysis indicated differences in these strains (the ITS sequences of all strains could be found in the Supplemental_datasets_S1.txt). All 22 isolates could be classified into 11 groups (Figure 1). The SHZ-4, SHZ-5, SHZ-6, SHZ-9, and SHZ-11 strains were distributed in the same branch, and have a close relationship with KJ696553.1. In these isolates, both SHZ-4 and SHZ-9 had a closer relationship with SHZ-5 in comparison to the others. Few of these isolates formed groups, such as SHZ-3, SHZ-15, and SHZ-20.

For further analysis the genctic relationship, all the strains were used to obtain the Nit mutant strains to do the VCG analysis. In total, 53 Nit mutants were obtained from 10 of 22 strains. The total numbers of Nit1, Nit M, and Nit 3 mutants were 39, 10, and 4, respectively (Supplemental_Table_S1.doc). The mutant strains were SHZ-2, SHZ-4, SHZ-5, SHZ-6, SHZ-8, SHZ-9, SHZ-11, SHZ-13, SHZ-18, and SHZ-21, respectively (Table 1). Three vegetative compatibility groups were found via mutant pairing. SHZ-4, SHZ-5, SHZ-6, SHZ-8, SHZ-9, and SHZ-11 isolates belong to one group (VCG1), which was consistent with the molecular phylogenetic tree analysis. This indicates that the isolates SHZ-4, SHZ-5, SHZ-6, SHZ-8, SHZ-9, and SHZ-11 had a closer relationship relative to the others.

\section{Pathogenicity differentiation test on the cotton hosts of $V$. dahliae strains}

The pathogenicity type could be divided into the following three categories: strong (average disease index value $\geq 40$ ), moderate $(25 \leq$ average disease index value $<40$ ), and weak (average disease index value $<25$ ). The strong pathogenicity category consisted of seven strains, which were SHZ-7, SHZ-9, SHZ-13, SHZ-16, SHZ-21, and SHZ-3 (Table 2). The average values of disease indexes of these isolates ranged from 51.20 to 43.52 . Half of the strains had moderate 
219 pathogenicity and the disease index values of which ranged from 35.48 to 26.24 . The strains that 220 belong to the moderate pathogenicity were the dominant population. SHZ-19, SHZ-10, SHZ-4, 221 SHZ-24, and SHZ-14 were classified as weakly pathogenic. The disease index values of the 222 weak pathogenicity group ranged from 21.57 to 18.36. According to both ITS analysis and 223 VCGs identification, the SHZ-4, SHZ-5, SHZ-6, SHZ-8, SHZ-9, and SHZ-11 had a close 224 relationship. However, these strains had different pathogenicity. This phenomenon illustrates that $225 V$. dahliae has a large variation at the population level.

\section{Transcriptome sequencing results for different pathogenicities of strains}

227

To investigate the reasons of the observed pathogenicity differentiation, the three strains 228 SHZ-4 (weak), SHZ-5 (moderate), and SHZ-9 (strong) that had a close relationship and different

229

230

231

232

233

234

235

236

237

238

239

240

241

242

243

244

245

246

247 pathogenicity, were used for transcriptome sequencing. Total RNA was extracted from the strains, and sequenced by the Illumina HiSeq 2500 platform. A total of $15.58 \mathrm{~Gb}$ of raw data were acquired. All clean reads of the three strains were used to assemble the transcriptome. A total of 19,700 unigenes, ranging from 201 to 16031 base pairs (bp) with 2,884 bp of N50, were obtained (Figure 2A). 67.4\% of the unigenes were annotated in at least one database (NR, NT, PFAM, CDD, KEGG, GO, Swissprot, TrEMBL, or KOG; Figure 2B). In SHZ-4_vs_SHZ-5, a total of 1908 differentially expressed genes (DEGs) were identified, 693 and 1215 of which were up- and down-regulated, respectively (Supplemental_Table_S2.xlsx). 1673 DEGs (808 upregulated and 865 downregulated) were found in SHZ-5_vs_SHZ-9. In SHZ-4_vs_SHZ-9, 610 unigenes were upregulated and 400 were downregulated (Figure 2C). According to Venn-iagram analysis, 146 unigenes were identified in all sample DEG data sets.

\section{Quantitative real-time-PCR validation of differentially expressed transcripts from RNA-}

\section{Seq}

Six unigenes were used to validate the expression patterns of the Illumina RNA-Seq results via qRT-PCR (Figure 3A and Supplemental_Table_S3.xlsx). These genes were extracellular trypsin protease (VTP1), SET domain-containing protein (VDAG_03353), dicarboxylic amino acid permease $\left(V D A G_{-}\right.$10467), rhamnogalacturonate lyase (VDAG_07119), ABC transporter (VDAG_05668), and cytochrome b2 (VDAG_07114). According to the qRT-PCR results, the expression levels of all six unigenes were lower in SHZ-4 than in SHZ-5 and SHZ-9. The 
248 transcription of extracellular trypsin protease, dicarboxylic amino acid permease, and $\mathrm{ABC}$ 249 transporter was ordered from low to high in the following: SHZ-4, SHZ-5, and SHZ-9, which is 250 consistent with their pathogenicity. Furthermore, the correlation between RNA-Seq and qRT251 PCR was evaluated using the foldchange value. As shown in Figure 3B, the qRT-PCR 252 measurements were moderately correlated with the RNA-Seq results $(r=0.77, \mathrm{R} 2=0.56)$, which 253 indicates that the RNA-Seq data were accurate and could be used for gene expression profile 254 analysis of the cold temperature defense response.

255 Gene ontology enrichment analysis of differentially expressed unigenes

256

257

258

259

260

261

262

263

264

265

266

267

268

269

270

271

272

273

274

275

276

All DEGs were analyzed via GO enrichment. The terms that were enriched in the SHZ4_vs_SHZ-5, SHZ-5_vs_SHZ-9, and SHZ-4_vs_SHZ-9 mainly focused on transport, membrane component, and relevant enzymes. In SHZ-4_vs_SHZ-5, SHZ-5_vs_SHZ-9, and SHZ4_vs_SHZ-9 DEG data sets, the transport term was most prevalent (Supplemental_Figures_S1, S2, and S3.pdf). These were involved in the organic acid transmembrane transporter activity, organic anion transmembrane transporter activity, substrate-specific transmembrane transporter activity, and organophosphate ester transport. Peptidase, pathogenesis, and hydrolase activity terms were enriched, all of which were related to pathogenicity. In SHZ-4_vs_ SHZ-5 and SHZ5_vs_ SHZ-9, these terms were mainly concentrated in the sugar metabolic process. However, the protein degradation, carbohydrate relative process, and pathogenesis terms were enriched in SHZ-4_vs_SHZ-9.

\section{Transport proteins were vital in the pathogenicity}

All unigenes on the transport were screened from the DEGs data set. There were 169 (9\% of the total DEGs), 109 (11\%), and 168 (10\%) unigenes that were found in SHZ-4_vs_SHZ-5, SHZ-4_vs_SHZ-9, and SHZ-5_vs_SHZ-9, respectively (Figure 4A). In SHZ-4_vs_SHZ-5, the transport proteins were mainly related to protein transporting $(28,16.57 \%$ of the total transport proteins), ion or inorganic salt transporting (30,17.75\%), carbohydrate transporting (47, 27.81\%), other organic transporting $(36,21.30 \%)$, and amino acid transporting $(18,10.65 \%)$. Among these unigenes, the numbers of the transport proteins were $26,19,40,30$, and 6 , respectively. The transport protein types, based on the transport substance type, were similar in SHZ-4_vs_SHZ-5, SHZ-4_vs_SHZ-9, and SHZ-5_vs_SHZ-9. However, the transport proteins on the protein 
277 transporting with the largest percentage in SHZ-4_vs_SHZ-9 differed from the SHZ-4_vs_SHZ2785 and SHZ-5_vs_SHZ-9, and both carbohydrate transport unigenes and others organic transport 279 unigenes provided the largest percentages in SHZ-4_vs_SHZ-5 and SHZ-5_vs_ SHZ-9, 280 respectively. It is well known that the transport of secreted proteins is related to vesicle transport. 281 Because the secreted proteins are related to pathogenicity, the unigenes involved in the vesicle 282 transport were analyzed. There were eight, nine, and five unigenes on the vesicle transport in the 283 SHZ-4_vs_SHZ-5, SHZ-4_vs_SHZ-9, and SHZ-5_vs_ SHZ-9 in total. According to the 284 expression level of these unigenes, only one unigene was screened from five unigenes in SHZ285 5_vs_SHZ-9.

286 There were twelve unigenes on the transport that extracted from the 146 unigenes data that 287 were identified in all SHZ-4_vs_SHZ-5, SHZ-5_vs_SHZ-9, and SHZ-4_vs_SHZ-9 DEG data. 288 In these unigenes, four unigenes that were Lactose permease, Alpha-glucosides permease, Sugar 289 transporter and L-fucose transporter had the function of transporting carbohydrates (Figure 4B). 290 The expression level of all the four unigens was highest in the SHZ-5. The expression level of 291 Lactose permease, Alpha-glucosides permease and Sugar transporter were lowest in the SHZ-4.

\section{Key genes responsible for pathogenicity differentiation}

293

294

295

296

297

298

299

300

301

302

303

304

305

306

Unigenes (146) that were identified in all SHZ-4_vs_SHZ-5, SHZ-5_vs_SHZ-9, and SHZ4_vs_SHZ-9 DEG data were used to analyze the expression mode (Figure 5 and Supplemental_Table_S4.xlsx). Based on the pathogenicity characteristic of the isolates, 19 unigenes were extracted from the 146 unigenes. The expression level of these 19 unigenes was lower in the SHZ-4 than in the SHZ-5 and SHZ-9. Among these 19 unigenes, the expression levels of seven unigenes showed the same order as the FPKM value from low to high in the SHZ-4, SHZ-5, and SHZ-9 isolates. These seven unigenes were metalloproteinase, integral membrane protein, inorganic pyrophosphatase, alkaline proteinase, dicarboxylic amino acid permease, phosphate-repressible phosphate permease, and extracellular trypsin protease. Metalloproteinase, alkaline proteinase, and extracellular trypsin protease belonged to the secretary proteins and can be used as virulence factors responsible for pathogenicity. The expression levels of the remaining 12 unigenes differed from those of the seven unigenes, and the expression levels of the unigenes in SHZ-5 was higher than in SHZ-9. Among the 12 unigenes, four had a function in host cell component degradation, i.e., Alpha-N- 
307 arabinofuranosidase A, polysaccharide deacetylase family protein, zinc carboxypeptidase A, and 308 rhamnogalacturonate lyase. Additional, four unigenes on the transport were filtered. These were 309 oligopeptide transporter, ABC transporter, quinate permease, and alpha-glucosides permease $310 \mathrm{MPH} 2 / 3$.

\section{Sugar-induced hyperosmotic tolerance positive correlation with the virulence}

312 The sugar-induced-hyperosmotic resistances of three $V$. dahliae isolates were analyzed by 313 changing the sugar consistence (Figure 6). The result illustrates that the stronger pathogenicity of $314 V$. dahliae yielded a higher resistance to hyperosmosis. The sugar tolerance limits of SHZ-4 and 315 SHZ-9 were $50 \mathrm{~g} / \mathrm{L}$ and $150 \mathrm{~g} / \mathrm{L}$, respectively, due to the growth inhibition of the hypha under 316 these conditions. However, the SHZ-9 could maintain normal growth at a sugar concentration of $317200 \mathrm{~g} / \mathrm{L}$. This suggests that a link should exist between pathogenicity and resistance to sugar 318 hyperosmotic stress. It is widely accepted that hyperosmosis of intercellular liquid could not only 319 leach moisture from the vessel into the hyperosmotic area via free diffusion, but could also help $320 V$. dahliae to scavenge nutrients from the host cell. To further investigate this, Zhongmian 35 321 was used to analyze the resistance to sugar-induced-hyperosmosis, which simulated the 322 processes caused by $V$. dahliae infection. Round leaf tissue was obtained by placing samples in 323 PDA medium with different sugar concentrations, similar to $V$. dahliae treatment. The result 324 indicates that brown spots were produced in the round leaf tissue 5 days later. Moreover, higher 325 sugar concentration led to a higher number of brown spots. Under conditions if high sugar 326 concentration, these brown spots were produced in the leaf tissue, unlike in low sugar concentration stress, where the brown spots were produced at the edge of the leaf tissue. This was similar to the symptoms caused by $V$. dahliae, where brown spots would emerge in the leaves of infected plants. Integration of the $V$. dahliae sugar-induced-hyperosmosis resistance with cotton symptoms during hyperosmosis conditions shows that the symptoms caused by $V$. dahliae could, at least, be partly attributed to the hyperosmosis produced by the degradation 332 process.

\section{Discussion}

\section{Cell-wall degrading enzymes underlying pathogenicity differentiation}

In this study, 22 isolates of $V$. dahliae were obtained from a cotton field. Through ITS and 
337 and SHZ-9. However, the isolates showed considerable differences in pathogenicity, with 338 virulence ranging from weak to strong with the order of SHZ-4, SHZ-5, and SHZ-9. A number 339 of genes were found via transcriptome sequencing. The genes related to protein and carbohydrate 340 degradation and transporting should be related to pathogenicity. The effectors as well as pectin 341 and cellulose degrading enzymes were important for pathogenicity (Buchner et al., 1982 and 342 1989; Davis et al., 1998; Mansoori et al., 2010; Meyer et al., 1994; Nachmias et al., 1985).

343 Genome comparison indicated that the $V$. dahliae genome contains more genes for cell-wall 344 degrading enzymes (CWDEs) than other fungi (Klosterman et al., 2011). Seven genes belonged 345 to the CWDEs in this study, which are likely responsible for the pathogenic differentiation of $V$. 346 dahliae. However, the genes of endopolygalacturonase $(P G)$, exopolygalacturonase $(P G X)$, and 347 glycoside hydrolase exerted no significant difference on the expression level. Accordingly, 348 although these genes are important for the pathogenicity (Liu et al., 2017a), they were not the 349 key effectors underlying pathogenic differentiation. A study reported evidence that the double 350 mutant of PG1 and PGX6 only reduced the virulence in tomato (Bravo Ruiz et al., 2016).

\section{Transport proteins implicated in pathogenicity differentiation}

352 Transport proteins played central role in the biological metabolism and regulation. Examples 353 were carbohydrate transport for the energy metabolism, ion transport for metal-enzymes and osmotic balance, and synthetic protein transport for types of metabolisms or regulation. A large number of unigenes on the transport (about $10 \%$ of the total DEGs) were screened out. These genes were implicated in many biological processes, for example protein transport, ion transport, carbohydrate transport, and other organic transport. Therefore, many transport genes related to the pathogenic differentiation of $V$. dahliae were not found in previous studies.

Almost all transport proteins were mediated by vesicular trafficking, which included secreted proteins and intracellular proteins. In this study based on the GO annotation, most of the transport proteins belonged to intracellular protein transport and only two genes were involved in the transport of secreted proteins. Many genes on the transport of secreted protein that are responsible for pathogenesis have been identified, e.g., MoSso1, MoSec22, and MoVamp7 in Magnaporthe oryzae (Song et al., 2010; Dou et al., 2011; Giraldo et al., 1996), FgSso1, FgVam7, and FgVps39 in Fusarium graminearum (Li et al., 2017), and VdSec22 and VdSsol in 
368 results indicate that the genes of CWDEs mainly resulted in different protein levels, which could 369 explain why few DEGs related to CWDEs were found. For the intracellular proteins transport, 370 these unigenes were implicated in pathogenic differentiation, and they seemed to be not directly 371 involved in pathogenicity. However, Vaclp, a vesicular transport protein for the intracellular 372 protein transport from Candida albicans, was required for both virulence and development 373 (Franke et al., 2006). Vaclp was important for metal ion resistance, such as $\mathrm{Cu}^{2+}, \mathrm{Zn}^{2+}$, and $\mathrm{Ni}^{2+}$ 374 (Franke et al., 2006). The result illustrates that the unigenes for the intracellular protein transport 375 were not directly related to pathogenicity. The gene Vps34 encodes a key protein for vacuolar 376 protein transport, which is involved in the secretion of aspartyl proteinases, metal ion resistance, 377 and fungistatic compounds (Kitanovic et al., 2005). A intracellular protein transported by the 378 vesicular transport system should be more closely related to the pathogenicity than the vesicular 379 transport genes on the intracellular protein transport (e.g., ion channels).

380 The carbohydrates, salts, and metal ions, and other organic molecules were most important 381 for $V$. dahliae nutrition. Most of the nutrition was absorbed via degradation of host-cells by the 382 CWDEs secreted by $V$. dahliae. With regard to the nutrient components, iron ions have been 383 studied in many pathogens. Iron is an essential nutrient for nearly all living organisms, including 384 eukaryotes and prokaryotes (Kaplan and Kaplan, 2009). Siderophores are most important for 385 obtaining iron from the extremely low available iron of host cells, which could contribute to the 386 iron homeostasis in the pathogen (Haas, 2003). However, the ABC-type $\mathrm{Fe}^{3+}$ transporter was only found in our study. The expression level of ABC-type Fe3+ transporter was highest in the SHZ-9 and lowest in the SHZ-4, which was positive correlation with the virulence of the three strains. Iron homeostasis could promote resistance to oxidative stress, through which the iron ion can affect the virulence of the pathogen (Gómez and Nosanchuk. 2003).

391 In addition to the nutrient consumption, transport proteins could play a role in other process. 392 With regard to virulence genes, the CWDEs could degrade the macromolecules of host cells into 393 small molecules. However, these small molecules greatly improved the osmotic pressure of the intercellular liquid. Therefore, improving the effectiveness of plant polygalacturonase-inhibiting 395 proteins (PGIPs) in reducing fungal polygalacturonase (PG) activity could enhance cotton 396 resistance to $V$. dahliae (Liu et al., 2018; Liu et al., 2017b). The winter embolism recovery of the walnut tree, where air bubbles in xylem vessels are cleared by an osmotic force produced via high-level PIP2 expression and the increase of sucrose from the conversion of starch-to-sugar 
399 corroborated this (Sakr et al., 2003). Likewise, the V.dahliae could affect the xylem vessels of 400 host plants to produce cavitations and starch hydrolysis, and vessel occlusion was considered 401 relevant to wilt symptoms (Trapero et al., 2018). Hyperosmosis of the intercellular liquid would 402 yield a detrimental effect on the surrounding of the host cell, which could lead to loss of moisture 403 and small molecules. The crop cultivars that could resist the $V$. dahliae was associated with 404 lignin biosynthesis, such as tomato ( $H u$ et al., 2019) and cotton ( $L i$ et al., 2019a). The 405 strengthening of cell walls might protect the host cell against the hyperosmotic harm. The effect 406 of hyperosmosis should be integrated into the symptoms caused by $V$. dahliae. This 407 physiological phenomenon indicated that $V$. dahliae had the ability to resist hyperosmosis and a 408 positive correlation will likely exist between virulence and the ability for hyperosmotic tolerance. 409 The $V d S s k 2$ gene, a mitogen-activated protein kinase kinase kinases (MAPKKKs) homologous, 410 was important in calcium signaling in fungi. Deletion of $V d S s k 2$ reduced the resistance to 411 osmotic stress and virulence of $V$. dahliae (Yu et al., 2019). Ectopic expression of GhSNAP33 412 enhancing the tolerance of yeast cells to oxidative and osmotic stresses and GhSNAP33-deficient 413 cotton being susceptible to $V$. dahliae infection (Wang et al., 2018b), illustrated the relation of 414 hyperosmotic resistance to Verticillium wilt. In Zhang et al., (2019b) research, the RD21-7 415 (responsive to desiccation 21-7) expression was induced by heat, cold and $V$. dahliae infection 416 and over-expression of GhRD21-7 enhanced resistance to $V$. dahliae in cotton. The GhRD21-7 417 gene conferring resistance to $V$. dahliae in cotton might be associates with the tolerance of 418 hyperosmotic stress, because of the cold stress inhibiting the water uptake by root and causing 419 the intracellular water losses. Therefore, preventing the intracellular water losses of host plants 420 would confer resistance to $V$. dahliae in theory. The hypothesis could be verified by the Wang et 421 al., (2019) research that the increased suberin of root cell wall improved the tolerance to $V$. 422 dahliae in Arabidopsis. It could be inferred that high resistance to hyperosmotic stresses could 423 confer resistance to $V$. dahliae. In Dong et al. (2019) research, gene silencing of a ATP-binding 424 cassette $(\mathrm{ABC})$ transporter $\mathrm{F}$ family member 5 (ABCF5) gene in Gossypium hirsutum reduced 425 the resistance to $V$. dahliae. The $A B C F 5$ gene might improve the tolerance to hyperosmotic 426 stresses to enhance the immunity of cotton to $V$. dahliae infection. Therefore, the resistance of $V$. 427 dahliae to hyperosmosis could affect its pathogenicity. In summary, transport-related DEGs 428 could be implicated in the pathogenicity differentiation of $V$. dahliae by changing the resistance 429 to hyperosmosis. 


\section{Conclusions}

432

433

434

435

436

437

438

439

440

441

442

443

444

445

446

447

448

449

450

451

452

453

454

455

456

457

458

459

460

461

462

463

464

Different $V$. dahliae strains possess different pathogenicity as a result of their large genetic variation. This variation was mainly encoded within CWDEs and transport related genes. It is widely accepted that the CWDEs determined the source of nutrients for $V$. dahliae and the organic substances of the host cells degraded by CWDEs would induce hyperosmosis of the intercellular liquid. Hyperosmosis not only induced dehydration and death of the host cell, but also contributed to the nutrient absorption by $V$. dahliae due to its high hyperosmosis tolerance of strains with that strong pathogenicity. In summary, both CWDEs and transport related genes are associated with the pathogenicity of $V$. dahliae. However, whether these transport related genes are associated with the hyperosmosis tolerance requires further research.

\section{Supplementary Materials:}

Supplementary files 1: Datasets S1. The ITS sequences of all strains (txt $7.20 \mathrm{~kb}$ )

Supplementary files 2: Table S1. The Nit mutant numbers and types (doc $33.5 \mathrm{~kb}$ )

Supplementary files 3: Table S2. DEGs identified in SHZ-4_vs_SHZ-5, SHZ-4_vs_SHZ-9, and SHZ-9_vs_SHZ-5 (ELSX 1.59 Mb)

Supplementary files 4: Table S3. Primer list and qRT-PCR data (ELSX $12.5 \mathrm{~kb}$ )

Supplementary files 5: Figure S1. Go enrichment analysis of SHZ-4_vs_SHZ-5 (PDF $7.19 \mathrm{~kb})$

Supplementary files 6: Figure S2. Go enrichment analysis of SHZ-4_vs_SHZ-9 (PDF $7.08 \mathrm{~kb}$ )

Supplementary files 7: Figure S3. Go enrichment analysis of SHZ-9_vs_SHZ-5 (PDF $7.07 \mathrm{~kb}$ )

Supplementary files 8: Table S4. Common DEGs identified in SHZ-4_vs_SHZ-5, SHZ4_vs_SHZ-9, and SHZ-9_vs_SHZ-5 (ELSX 63.7 kb)

Author Contributions: Jin Li, Wenwen Xia, and Juan Pei isolated the strains and conducted the ITS analysis. Jin Li, Juan Pei, Wenwen Xia, Wenhui Tian, and Yuanyuan Liu performed pathogenicity assays, VCGs assays, and transcriptome analyses. Yuanyuan Liu, Wenwen Xia, and Fengfeng Cheng performed qRT-PCR experiments and hyperosmosis resistance experiments of $V$. dahliae and cotton leaves. Jin Li wrote the manuscript. Zhongping Lin, Jianbo Zhu, and Aiying Wang guided the study and approved the manuscript for publication. All authors read and approved the final manuscript.

Funding: This work was funded by the transgenic major project of cotton (2016ZX08011-004) and the National Natural Science Foundation of China (31360053).

Conflicts of Interest: The authors declare there are no competing interests.

\section{Data Availability:}


465 All sequencing data generated for this work have been deposited in the NCBI Gene Expression Omnibus (GEO) under accession number GSE131467.

467

\section{References}

469

470

471

472

473

474

475

476

477

478

479

480

481

482

483

484

485

486

487

488

489

490

491

492

493

494

495

496

497

498

499

500

501

502

503

504

505

506

507

508

509

510

Amyotte SG, Tan X, Pennerman K, Jimenez-Gasco Mdel M, Klosterman SJ, Ma LJ, Dobinson KF, Veronese P. 2012. Transposable elements in phytopathogenic Verticillium spp.: insights into genome evolution and inter- and intra-specific diversification. $B M C$ Genomics. 13: 314 DOI 10.1186/1471-2164-13-314.

Audic S, Claverie JM. 1997. The significance of digital gene expression profiles. Genome Research 7: 986-95 DOI 10.1101/gr.7.10.986.

Bao JR, Katan J, Shabi E, Katan T. 1998. Vegetative-compatibility groups in Verticillium dahliae from Israel. European Journal of Plant Pathology 104: 263-269 DOI 10.1023/a:1008696312859.

Baroudy F, Putman AI, Habib W, Puri KD, Subbarao KV, Nigro F. 2019. Genetic Diversity of Verticillium dahliae Populations From Olive and Potato in Lebanon. Plant Dis 103:656667.

Bravo Ruiz G, Di Pietro A, Roncero MI. 2016. Combined action of the major secreted exoand endopolygalacturonases is required for full virulence of Fusarium oxysporum. Molecular Plant Pathology 17: 339-53 DOI 10.1111/mpp.12283.

Buchner V, Burstein Y, Nachmias A. 1989. Comparison of Verticillium dahliae produced phytotoxic peptides purified from culture fluids and infected potato stems. Physiological and Molecular Plant Pathology 35: 253-269 DOI 10.1016/0885-5765(89)90055-6.

Buchner V, Nachmias A, Burstein Y. 1982. Isolation and partial characterization of a phytotoxic glycopeptide from a proteinlipopolysaccharide complex produced by a potato isolate of Verticillium dahliae. Febs Letters 138: 261-264 DOI 10.1016/00145793(82)80456-0.

Cai YF, He XH, Mo JC, Sun Q, Yang JP, Liu JG. 2009. Molecular research and genetic engineering of resistance to Verticillium wilt in cotton: A review. African Journal of Biotechnology 8: 7363-7372

Chen JY, Xiao HL, Gui YJ, Zhang DD, Li L, Bao YM, Dai XF. 2016. Characterization of the Verticillium dahliae Exoproteome Involves in Pathogenicity from Cotton-Containing Medium. Frontiers In Microbiology 7: 1709. DOI 10.3389/fmicb.2016.01709.

Chen JY, Liu C, Gui YJ, Si KW, Zhang DD, Wang J, Short DPG, Huang JQ, Li NY, Liang Y, Zhang WQ, Yang L, Ma XF, Li TG, Zhou L, Wang BL, Bao YM, Subbarao KV, Zhang GY, Dai XF. 2017. Comparative genomics reveals cotton-specific virulence factors in flexible genomic regions in Verticillium dahliae and evidence of horizontal gene transfer from Fusarium. New Phytologist 217: 756-770 DOI 10.1111/nph.14861.

Chu J, Li WF, Cheng W, Lu M, Zhou KH, Zhu HQ, Li FG, Zhou CZ. 2015. Comparative analyses of secreted proteins from the phytopathogenic fungus Verticillium dahliae in response to nitrogen starvation. Biochimica Et Biophysica Acta-Biomembranes 1854: 437448 DOI 10.1016/j.bbapap.2015.02.004

Daayf F, Nicole M, Geiger JP. 1995. Differentiation of Verticillium dahliae populations on the basis of vegetative compatibility and pathogenicity on cotton. European Journal of Plant Pathology 101: 69--79 DOI 10.1007/BF01876095.

Davis DA, Low PS, Heinstein P. 1998. Purification of a glycoprotein elicitor of phytoalexin formation from Verticillium dahliae. Physiological and Molecular Plant Pathology 52:

Peer] reviewing PDF | (2019:05:37736:2:0:NEW 8 Oct 2019) 
511

512

513

514

515

516

517

518

519

520

521

522

523

524

525

526

527

528

529

530

531

532

533

534

535

536

537

538

539

540

541

542

543

544

545

546

547

548

549

550

551

552

553

554

555

556

259-273 DOI 10.1006/pmpp.1998.0150.

de Jonge R, Bolton MD, Kombrink A, van den Berg GC, Yadeta KA, Thomma BP. 2013. Extensive chromosomal reshuffling drives evolution of virulence inan asexual pathogen. Genome Research 23: 1271 -1282 DOI 10.1101/gr.152660.112.

Dong Q, Magwanga RO, Cai X, Lu P, Nyangasi Kirungu J, Zhou Z, Wang X, Wang X, Xu Y, Hou Y, Wang K, Peng R, Ma Z, Liu F. 2019. RNA-Sequencing, Physiological and RNAi Analyses Provide Insights into the Response Mechanism of the ABC-Mediated Resistance to Verticillium dahliae Infection in Cotton. Genes 10: 110. DOI 10.3390/genes10020110.

Dou X, Wang Q, Qi Z, Song W, Wang W, Guo M, Zhang H, Zhang Z, Wang P, Zheng X. 2011. MoVam 7, A conserved SNARE involved in vacuole assembly, is required for growth, endocytosis, ROS accumulation, and pathogenesis of Magnaporthe oryzae. PLoS One 6: e16439 DOI 10.1371/journal.pone.0016439.

Dung JKS, Knaus BJ, Fellows HS, Grünwald NJ, Vining KJ. 2019. Genetic Diversity of Verticillium dahliae Isolates from Mint Detected with Genotyping by Sequencing. Phytopathology DOI 10.1094/PHYTO-12-18-0475-R.

Fradin EF, Thomma BP. 2006. Physiology and molecular aspects of Verticillium wilt diseases caused by V. Dahliae and V. alboatrum. Molecular Plant Pathology 7:71-86 DOI 10.1111/j.1364-3703.2006.00323.x.

Franke K, Nguyen M, Härtl A, Dahse HM, Vogl G, Würzner R, Zipfel PF, Künkel W, Eck R. 2006. The vesicle transport protein Vaclp is required for virulence of Candida albicans. Microbiology 152: 3111-3121 DOI 10.1099/mic.0.29115-0.

Giraldo MC, Dagdas YF, Gupta YK, Mentlak TA, Yi M, Martinez-Rocha AL, Saitoh H, Terauchi R, Talbot NJ, Valent B. 2013. Two distinct secretion systems facilitate tissue invasion by the rice blast fungus Magnaporthe oryzae. Nature Communications 4: 1996 DOI 10.1038/ncomms2996.

Glass NL, Schmoll M, Cate JH, Coradetti S. 2013. Plant cell wall deconstruction by ascomycete fungi. Annual Review of Microbiology 67: 477-498 DOI 10.1146/annurevmicro-092611-150044.

Gómez BL, Nosanchuk JD. 2003. Melanin and fungi. Curr. Opin. Current Opinion in Infectious Diseases 16: 91-96 DOI 10.1097/01.aco.0000065076.06965.04.

Göre ME. 2007. Vegetative compatibility and pathogenicity of Verticillium dahliae isolates from the aegean region of Turkey. Phytoparasitica 35: 222-231 DOI 10.1007/bf02981154.

Haas H. 2003. Molecular genetics of fungal siderophore biosynthesis and uptake: the role of siderophores in iron uptake and storage. Applied Microbiology and Biotechnology 62: 316330 DOI 10.1007/s00253-003-1335-2.

Hu X, Puri KD, Gurung S, Klosterman SJ, Wallis CM, Britton M, Durbin-Johnson B, Phinney B, Salemi M, Short DPG, Subbarao KV. 2019. Proteome and metabolome analyses reveal differential responses in tomato -Verticillium dahliae-interactions. $J$ Proteomics 207:103449 DOI 10.1016/j.jprot.2019.103449.

Huisman OC. 1982. Interrelations of root growth dynamics to epidemiology of root-invading fungi. Annual Review of Phytopathology. 20: 303-327. DOI 10.1146/annurev.py.20.090182.001511.

Joaqium TR, Rowe RC. 1990. Reassessment of Vegetative-compatibility relationships among strains of Vertivillium dahliae using nitrate-nonutilizing mutants. Phytopathology 80: 11601166 DOI 10.1094/Phyto-80-1160. 
557

558

559

560

561

562

563

564

565

566

567

568

569

570

571

572

573

574

575

576

577

578

579

580

581

582

583

584

585

586

587

588

589

590

591

592

593

594

595

596

597

598

599

600

601

Joaqium TR, Rowe RC. 1991. Vegetative compatibility and virulence of strains of Verticillium dahliae from soil and potato plants. Phytopathology 81: 552-558 DOI 10.1094/Phyto-81552.

Kaplan CD, Kaplan J. 2009. Iron acquisition and transcriptional regulation. Chemical Reviews 109: 4536-4552 DOI 10.1021/cr9001676.

King BC, Waxman KD, Nenni NV, Walker LP, Bergstrom, Gibson DM. 2011. Arsenal of plant cell wall degrading enzymes reflects host preference among plant pathogenic fungi. Biotechnology for Biofuels 4: 4-16 DOI 10.1186/1754-6834-4-4.

Kitanovic A, Nguyen M, Vogl G, Hartman A, Gunther J, Wurzner R, Kunkel W, Wolfl S, Eck R. 2005. Phosphatidylinositol 3-kinaseVPS34 of Candida albicans is involved in filamentous growth, Saps secretion, and intracellular detoxification. FEMS Yeast Research 5: 431-9 DOI 10.1016/j.femsyr.2004.11.005.

Klimes A, Dobinson KF. 2006. A hydrophobin gene, VDH1, is involved in microsclerotial development and spore viability in the plant pathogenVerticillium dahliae. Fungal Genetics and Biology 43: 283-294. DOI 10.1016/j.fgb.2005.12.006.

Klosterman SJ, Atallah ZK, Vallad GE, Subbarao KV. 2009. Diversity, pathogenicity, and management of Verticillium species. Annual Review of Phytopathology 47:39-62 DOI 10.1146/annurev-phyto-080508-081748.

Klosterman SJ, Subbarao KV, Kang S, Veronese P, Gold SE, Thomma BP, Chen Z, Henrissat, B, Lee YH, Park J, Garcia-Pedrajas MD, Barbara DJ, Anchieta A, de Jonge R, Santhanam P, Maruthachalam K, Atallah Z, Amyotte SG, Paz Z, Inderbitzin P, Hayes RJ, Heiman DI, Young S, Zeng Q, Engels R, Galagan J, Cuomo CA, Dobinson KF, Ma LJ. 2011. Comparative genomics yields insights into niche adaptation of plant vascular wilt pathogens. PLoS Pathogens 7: e1002137 DOI 10.1371/journal.ppat.1002137.

Korolev N, Peres-Artes E, Bejarano-Alcazar J, Rodriguez-Jurado D, Katan J, Katan T, Jimenez-Diaz RM. 2001. Comparative study of genetic diversity and pathogenicity among populations of VerticiUium dahliae from cotton in Spain and Israel. European Journal of Plant Pathology 107: 443-456 DOI 10.1023/a:1011212426447.

Kubicek CP, Starr TL, Glass NL. 2014. Plant cell wall-degrading enzymes and their secretion in plant-pathogenic fungi. Annual Review of Phytopathology 52: 427-451 DOI 10.1146/annurev-phyto-102313-045831.

Li B, Liu L, Li Y, Dong X, Zhang H, Chen H, Zheng X, Zhang Z. 2017. The FgVps39FgVam7-FgSsol Complex mediates vesicle trafficking and is important for the development and virulence of Fusarium graminearum. Molecular Plant-Microbe Interactions 30: 410-422 DOI 10.1094/MPMI-11-16-0242-R.

Li C, He Q, Zhang F, Yu J, Li C, Zhao T, Zhang Y, Xie Q, Su B, Mei L, Zhu S, Chen J. 2019a. Melatonin enhances cotton immunity to Verticillium wilt via manipulating lignin and gossypol biosynthesis. Plant J DOI 10.1111/tpj.14477.

Li PT, Rashid MHO, Chen TT, Lu QW, Ge Q, Gong WK, Liu AY, Gong JW, Shang HH, Deng XY, Li JW, Li SQ, Xiao XH, Liu RX, Zhang Q, Duan L, Zou XY, Zhang Z, Jiang X, Zhang Y, Peng RH, Shi YZ, Yuan YL. 2019b. Transcriptomic and biochemical analysis of upland cotton (Gossypium hirsutum) and a chromosome segment substitution line from $G$. hirsutum $\times$ G. barbadense in response to Verticillium dahliae infection. BMC Plant Biol. 19:19. DOI 10.1186/s12870-018-1619-4. 
602

603

604

605

606

607

608

609

610

611

612

613

614

615

616

617

618

619

620

621

622

623

624

625

626

627

628

629

630

631

632

633

634

635

636

637

638

639

640

641

642

643

644

645

646

647

Liu N, Ma X, Sun Y, Hou Y, Zhang X, Li F. 2017a. Necrotizing Activity of Verticillium dahliae and Fusarium oxysporum f. sp. vasinfectum Endopolygalacturonases in Cotton. Plant Dis 101:1128-1138.

Liu N, Sun Y, Wang P, Duan H, Ge X, Li X, Pei Y, Li F, Hou Y. 2018. Mutation of key amino acids in the polygalacturonase-inhibiting proteins CkPGIP1 and GhPGIP1 improves resistance to Verticillium wilt in cotton. Plant J 96:546-561. DOI 10.1111/tpj.14048.

Liu N, Zhang X, Sun Y, Wang P, Li X, Pei Y, Li F, Hou Y. 2017b. Molecular evidence for the involvement of a polygalacturonase-inhibiting protein, GhPGIP1, in enhanced resistance to Verticillium and Fusarium wilts in cotton. Sci Rep 7:39840. DOI 10.1038/srep39840.

Lopez MA, Bannenberg G, Castresana C. 2008. Controlling hormone signaling is a plant and pathogen challenge for growth and survival. Curr. Opin. Plant Biol. 11:420-427. DOI 10.1016/j.pbi.2008.05.002.

Luo X, Xie, Dong J, Yang X, Sui A. 2014. Interactions between Verticillium dahliae and its host: vegetative growth, pathogenicity, plant immunity. Applied Microbiology and Biotechnology 98: 6921 -6932 DOI 10.1007/s00253-014-5863-8.

Rowe RC. 1995. Recent progress in understanding relationships between Verticillium species and subspecific groups. Phytoparasitica 23: 31-38 DOI 10.1007/bf02980394.

Mansoori B, Milton JM, Smith CJ. 2010. Isolation and partial purification of a phytotoxin related to pathogenic Verticillium species. Journal of Phytopathology 143: 33-36 DOI 10.1111/j.1439-0434.1995.tb00196.x.

Meyer R, Slater V, Dubery LA. 1994. A phytotoxic proteinlipopolysccharide complex produced by Verticillium dahlia. Phytochemistry 35: 1449-1453 DOI 10.1016/S00319422(00)86872-7.

Nachmias A, Buchner V, Burstein Y. 1985. Biological and immunochemical characterization of a low molecular weight phytotoxin isolated from a protein-lipopolysaccharide complex produced by a potato isolate of Verticillium dahliae Kleb. Physiological Plant Pathology 26: 43-55 DOI 10.1016/0048-4059(85)90029-3.Sanei SJ, Okhovvat SM, Javan-Nikkhah M, Saremi H. 2005. Vegetative compatibility and pathogenicity of Verticillium dahliae Kleb. isolates from Olive in Iran. Communications In Agricultural and Applied Biological Sciences 70: $323-5$

Pieterse CM, Leon-Reyes A, Van der Ent S, Van Wees SC. 2009. Networking by smallmolecule hormones in plant immunity. Nat. Chem. Biol. 5:308-316. DOI 10.1038/nchembio. 164.

Pieterse CM, van Wees SC, van Pelt JA, Knoester M, Laan R, Gerrits H, Weisbeek PJ, van Loon LC. 1998. A novel signaling pathway controlling induced systemic resistance in Arabidopsis. Plant Cell 10:1571-1580. DOI 10.1105/tpc.10.9.1571.

Sakr S, Alves G, Morillon R, Maurel K, Decourteix M, Guilliot A, Fleurat-Lessard P, Julien JL, Chrispeels MJ. 2003. Plasma Membrane Aquaporins Are Involved in Winter Embolism Recovery in Walnut Tree. Plant Physiology 133: 630-41 DOI 10.1104/pp.103.027797.

Schnathorst WC, Mathre DE. 1966. Host range and differentiation of a severe form of Verticillium albo-atrum in cotton. Phytopathology 56: 1155-1161

Song W, Dou X, Qi Z, Wang Q, Zhang X, Zhang H, Guo M, Dong S, Zhang Z, Wang P, Zheng X. 2010. R-SNARE homolog MoSec22 is required for conidiogenesis, cell wall integrity, and pathogenesis of Magnaporthe oryzae. PLoS One 395: e13193 DOI 10.1371/journal.pone.0013193.

Peer) reviewing PDF | (2019:05:37736:2:0:NEW 8 Oct 2019) 
648

649

650

651

652

653

654

655

656

657

658

659

660

661

662

663

664

665

666

667

668

669

670

671

672

673

674

675

676

677

678

679

680

681

682

683

684

685

686

687

688

689

690

691

692

693

Sun Q, Jiang H, Zhu X, Wang W, He X, Shi Y, Yuan Y, Du X, Cai Y. 2013. Analysis of seaisland cotton and upland cotton in response to Verticillium dahliae infection by RNA sequencing. BMC Genomics 14:852.

Trapero C, Alcántara E, Jiménez J, Amaro-Ventura MC, Romero J, Koopmann B, Karlovsky P, von Tiedemann A, Pérez-Rodríguez M, López-Escudero FJ. 2018. Starch Hydrolysis and Vessel Occlusion Related to Wilt Symptoms in Olive Stems of Susceptible Cultivars Infected by Verticillium dahliae. Front Plant Sci 9:72. DOI 10.3389/fpls.2018.00072.

Tzima AK, Paplomatas EJ, Rauyaree P, Ospina-Giraldo MD, Kang S. 2011. VdSNF1, the sucrose nonfermenting protein kinase gene of Verticillium dahliae, is required for virulence and expression of genes involved in cell-wall degradation. Molecular Plant-Microbe Interactions 24: 129-142 DOI 10.1094/MPMI-09-09-0217.

Vlot AC, Dempsey DA, Klessig DF. 2009. Salicylic acid, a multifaceted hormone to combat disease. Annu Rev. Phytopathol. 47:177-206. DOI 10.1146/annurev.phyto.050908.135202.

Wang J, Tian L, Zhang DD, Short DPG, Zhou L, Song SS, Liu Y, Wang D, Kong ZQ, Cui WY, Ma XF, Klosterman SJ, Subbarao KV, Chen JY, Dai XF. 2018a. SNAREEncoding Genes VdSec22 and VdSso1 Mediate Protein Secretion Required for Full Virulence in Verticillium dahliae. Molecular Plant-Microbe Interactions 31: 651-664 DOI 10.1094/MPMI-12-17-0289-R.

Wang G, Xu J, Li L, Guo Z, Si Q, Zhu G, Wang X, Guo W. 2019. GbCYP86A1-1 from Gossypium barbadense positively regulates defence against Verticillium dahliae by cell wall modification and activation of immune pathways. Plant Biotechnol $J$ DOI 10.1111/pbi.13190.

Wang P, Sun Y, Pei Y, Li X, Zhang X, Li F, Hou Y. 2018b. GhSNAP33, a t-SNARE Protein From Gossypium hirsutum, Mediates Resistance to Verticillium dahliae Infection and Tolerance to Drought Stress. Front Plant Sci 9:896. DOI 10.3389/fpls.2018.00896.

Yang J, Wang G, Ke H, Zhang Y, Ji L, Huang L, Zhang C, Wang X, Ma Z. 2019. Genomewide identification of cyclophilin genes in Gossypium hirsutum and functional characterization of a CYP with antifungal activity against Verticillium dahliae. BMC Plant Biol 19:272. DOI 10.1186/s12870-019-1848-1.

Yu J, Li T, Tian L, Tang C, Klosterman SJ, Tian C, Wang Y. 2019. Two Verticillium dahliae MAPKKKs, VdSsk2 and VdSte11, Have Distinct Roles in Pathogenicity, Microsclerotial Formation, and Stress Adaptation. mSphere 4: e00426-19. doi: 10.1128/mSphere.00426-19.

Zhang J, Zhang Y, Yang J, Kang L, EloRM AM, Zhou H, Zhao J. 2019a. The $\alpha-1,6-$ mannosyltransferase $\mathrm{VdOCH} 1$ plays a major role in microsclerotium formation and virulence in the soil-borne pathogen Verticillium dahliae. Fungal Biol 123: 539-546.

Zhang S, Xu Z, Sun H, Sun L, Shaban M, Yang X, Zhu L. 2019b. Genome-Wide Identification of Papain-Like Cysteine Proteases in Gossypium hirsutum and Functional Characterization in Response to Verticillium dahliae. Front Plant Sci 10:134. DOI 10.3389/fpls.2019.00134.

Zhang Z, Wang P, Luo X, Yang C, Tang Y, Wang Z, Hu G, Ge X, Xia G, Wu J. 2019c. Cotton plant defence against a fungal pathogen is enhanced by expanding BLADE-ONPETIOLE1 expression beyond lateral-organ boundaries. Commun Biol 2:238. DOI 10.1038/s42003-019-0468-5.

Zhu HQ, Feng ZL, Li ZF, Shi YQ, Zhao LH. 2013. Characterization of two fungal isolates from cotton and evaluation of their potential for biocontrol of Verticillium wilt of cotton.

Peer) reviewing PDF | (2019:05:37736:2:0:NEW 8 Oct 2019) 
694 Journal of Phytopathology 161: 70-77 DOI 10.1111/jph.12027 
Figure 1

\section{The phylogenetic tree analysis based on the ITS sequences of $V$. dahliae}

no

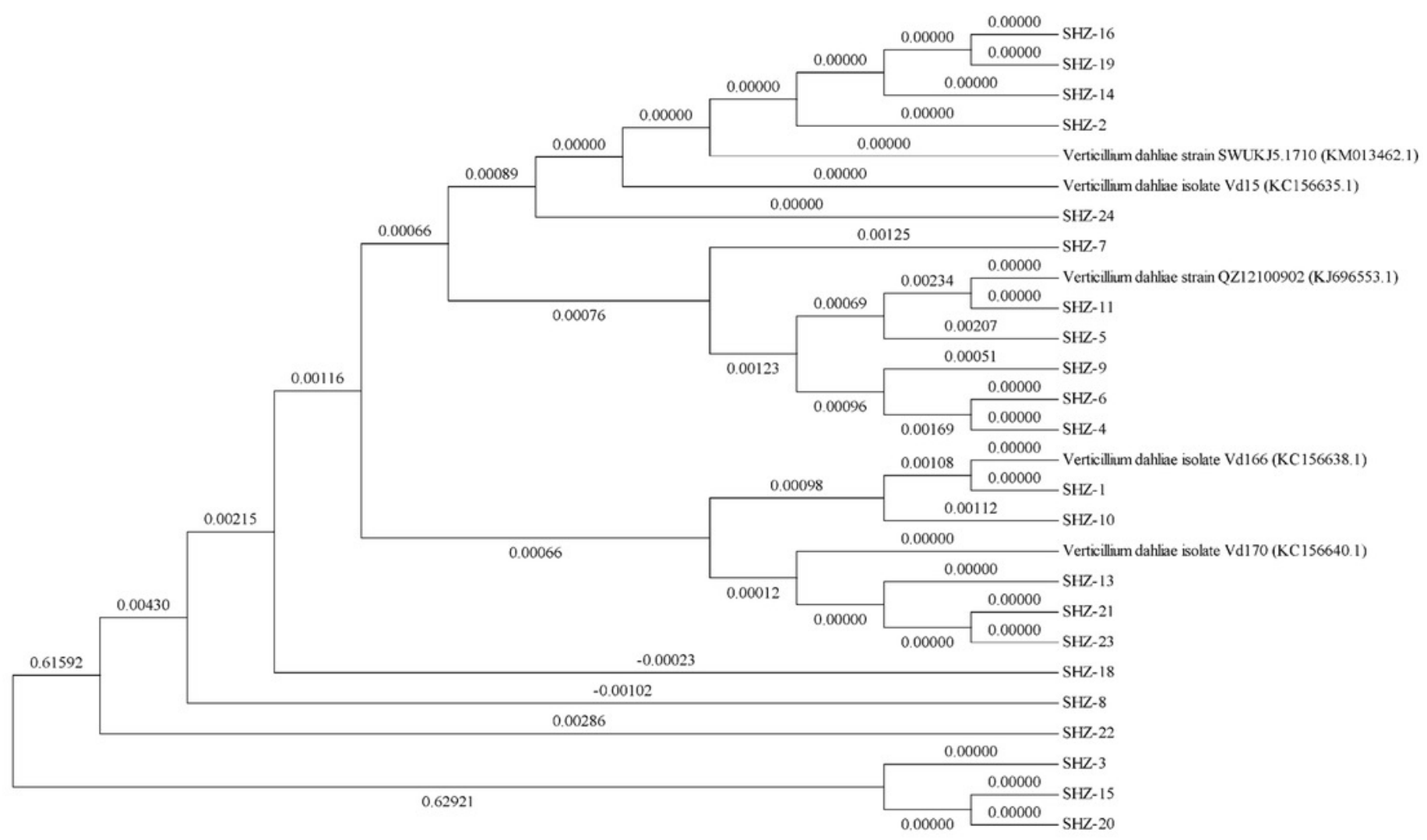


Figure 2

Transcriptome assembly and annotation statistic

(A) the unigenes length distribution satistic after transcriptome assembly; (B) the statistics of unigenes annotation in different databases; (C) Venn diagrams of comparisons between the DEGs of the strains. 
A

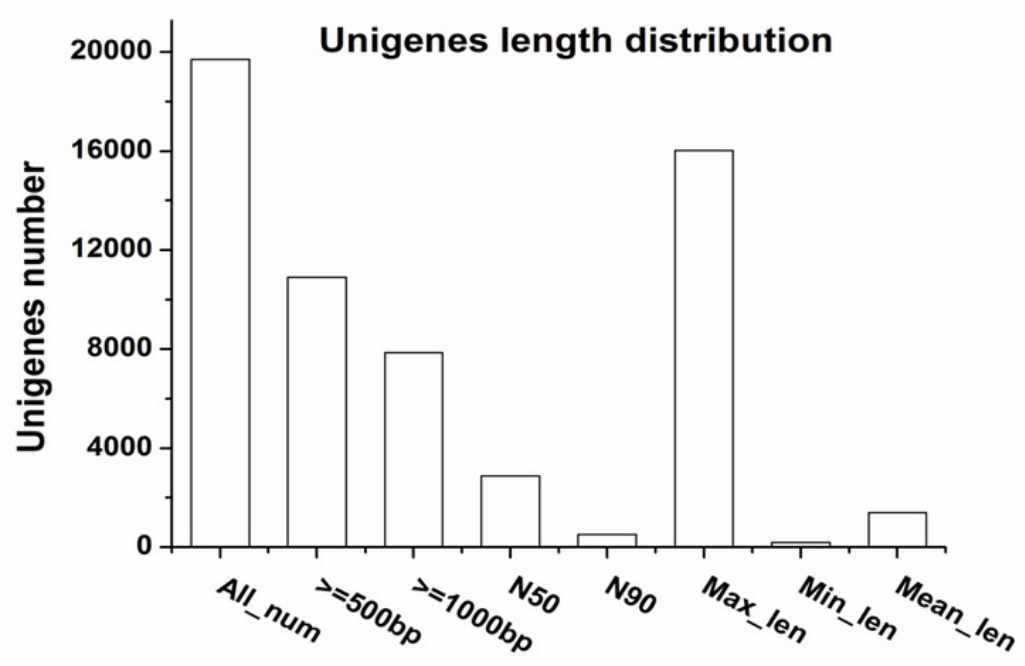

B

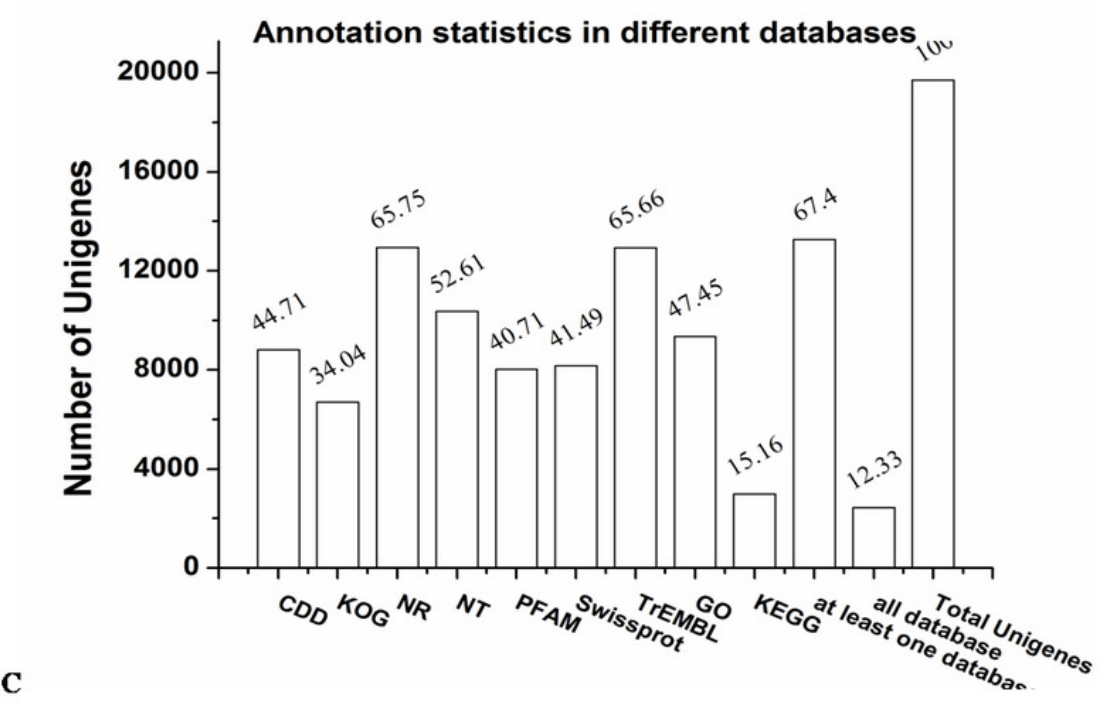

SHZ-4_vs_SHZ-5 SHZ-4_vs_SHZ-9

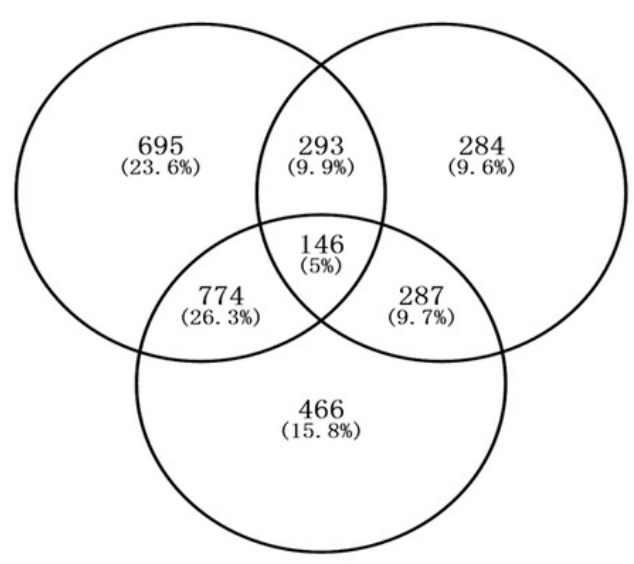

SHZ-5_vs_SHZ-9 
Figure 3

The unigenes expression level analyzed by the qRT-PCR

(A) the relative expression level of the unigenes in different strains; (B) correlation analysis between RNA-Seq and qRT-PCR

A

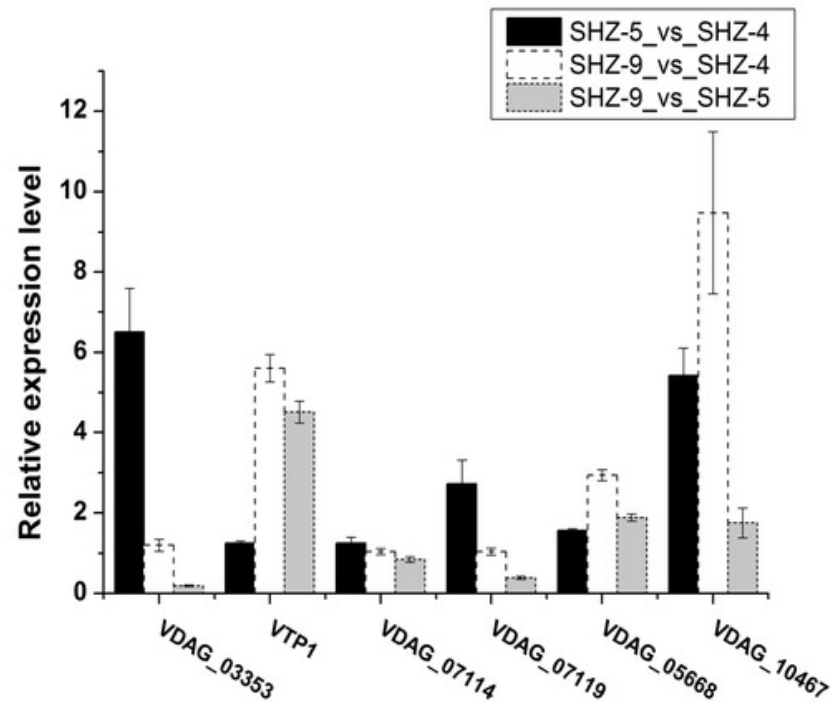

B

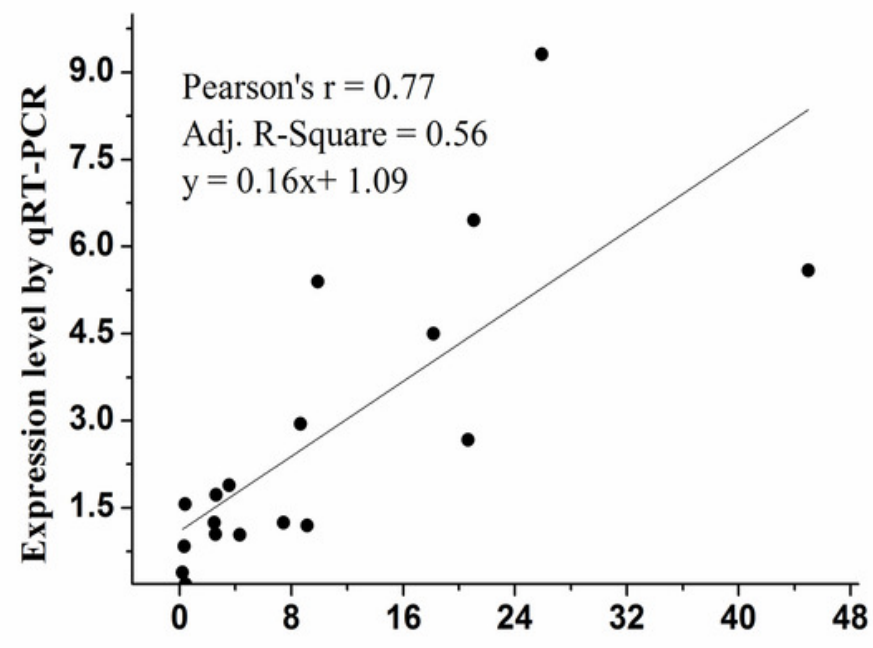

Expression level by RNA-Seq (Foldchange) 
Figure 4

Unigenes on different substance transport

(A) Unigenes proportion on different substance transport. (B) Expression level of genes on the carbonhydrates. 
A

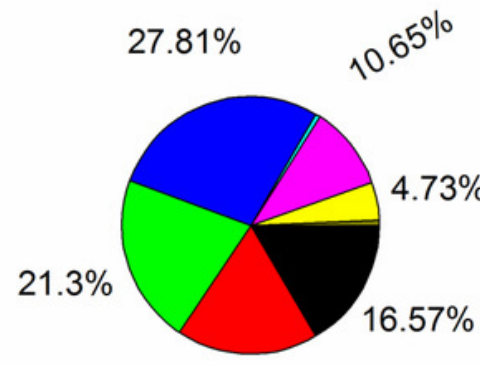

$17.75 \%$

SHZ-4_vs_SHZ-5

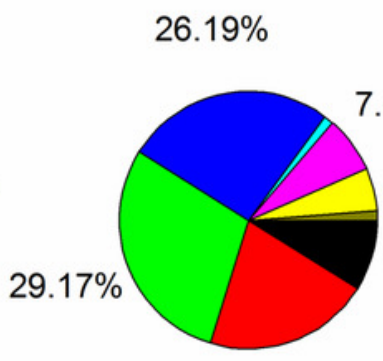

$20.83 \%$

SHZ-5_vs_SHZ-9
$21.1 \% \quad 3.67 \%$

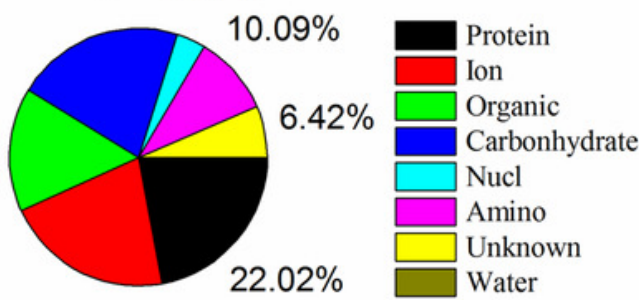

$21.1 \%$

SHZ-4_vs_SHZ-9

B
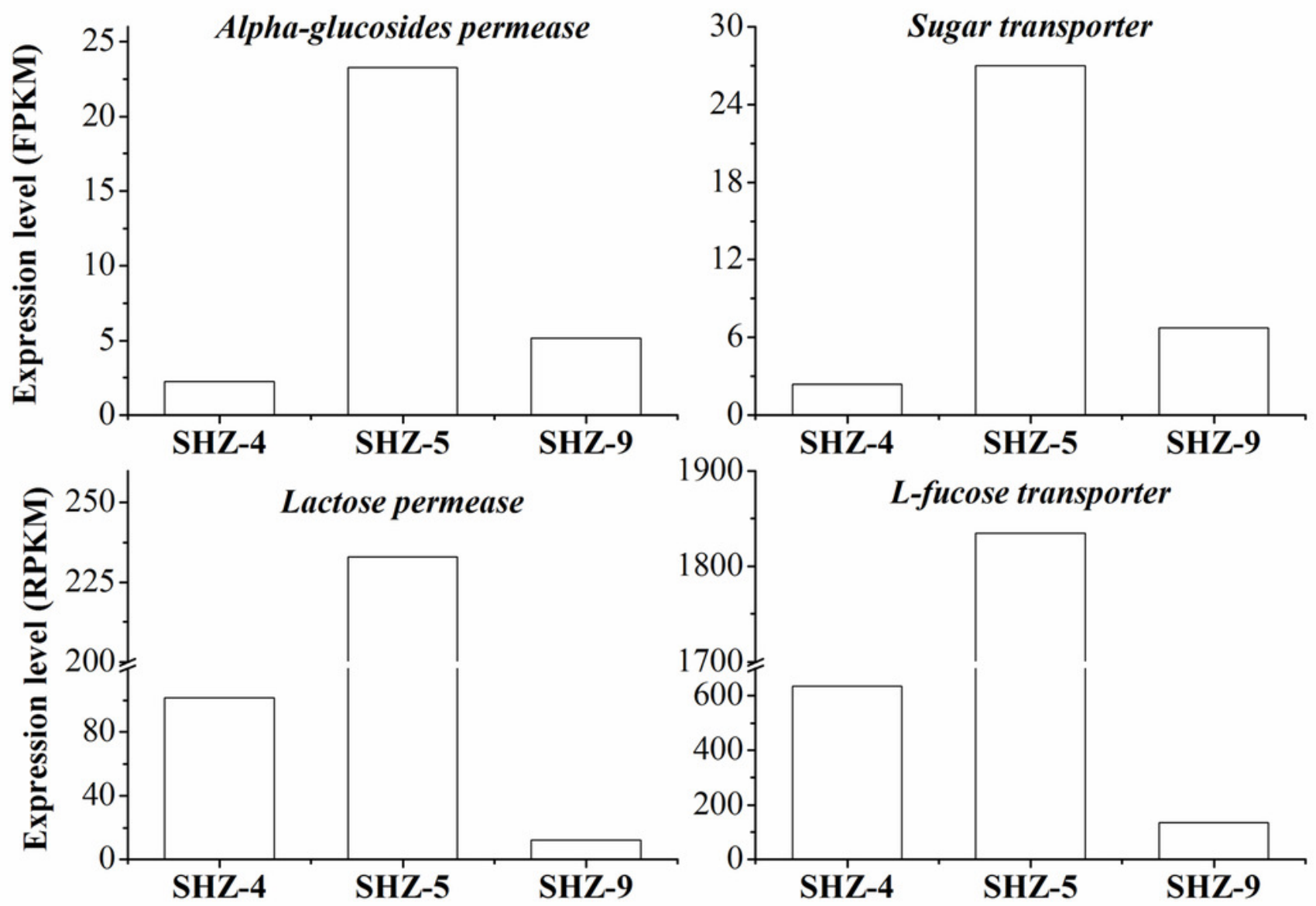


\section{Figure 5}

Expression levels of 19 unigenes selected according to the pathogenicities of SHZ-4, SHZ-5, and SHZ-9

The FPKM value was used as expression level value
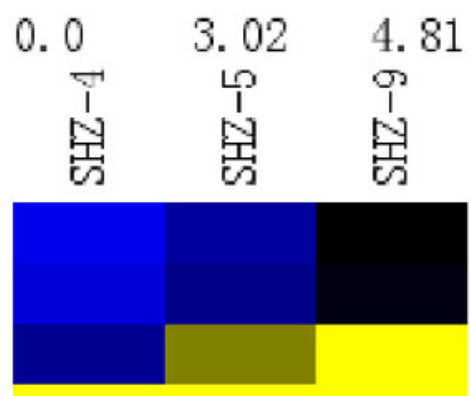

Integral membrane protein

Inorganic pyrophosphatase

Dicarboxylic amino acid permease

Phosphate-repressible phosphate permease

Transport relative

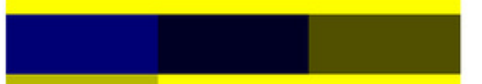

Metalloproteinase

Alkaline proteinase

Extracellular trypsin protease

Zinc carboxypeptidase $A$

Alpha- $N$-arabinofuranosidase $A$

Polysaccharide deacetylase family protein

Rhamnogalacturonate lyase

Oligopeptide transporter

Quinate permease

$A B C$ transporter

Alpha-glucosides permease $\mathrm{MPH} 2 / 3$

Putative uncharacterized protein

SET domain-containing protein

Putative uncharacterized protein

Cytochrome b2

Transport relative

Cell wall degradation

Proteolysis

Cell wall degradion 


\section{Figure 6}

Identification of $\mathrm{V}$. dahliae strains and cotton leaves that are resistant to sugar-inducedhyperosmosis

(A) the sugar-induced-hyperosmotic resistances of three $\mathrm{V}$. dahliae isolates; (B) the cotton leaf resistance to sugar-induced-hyperosmosis

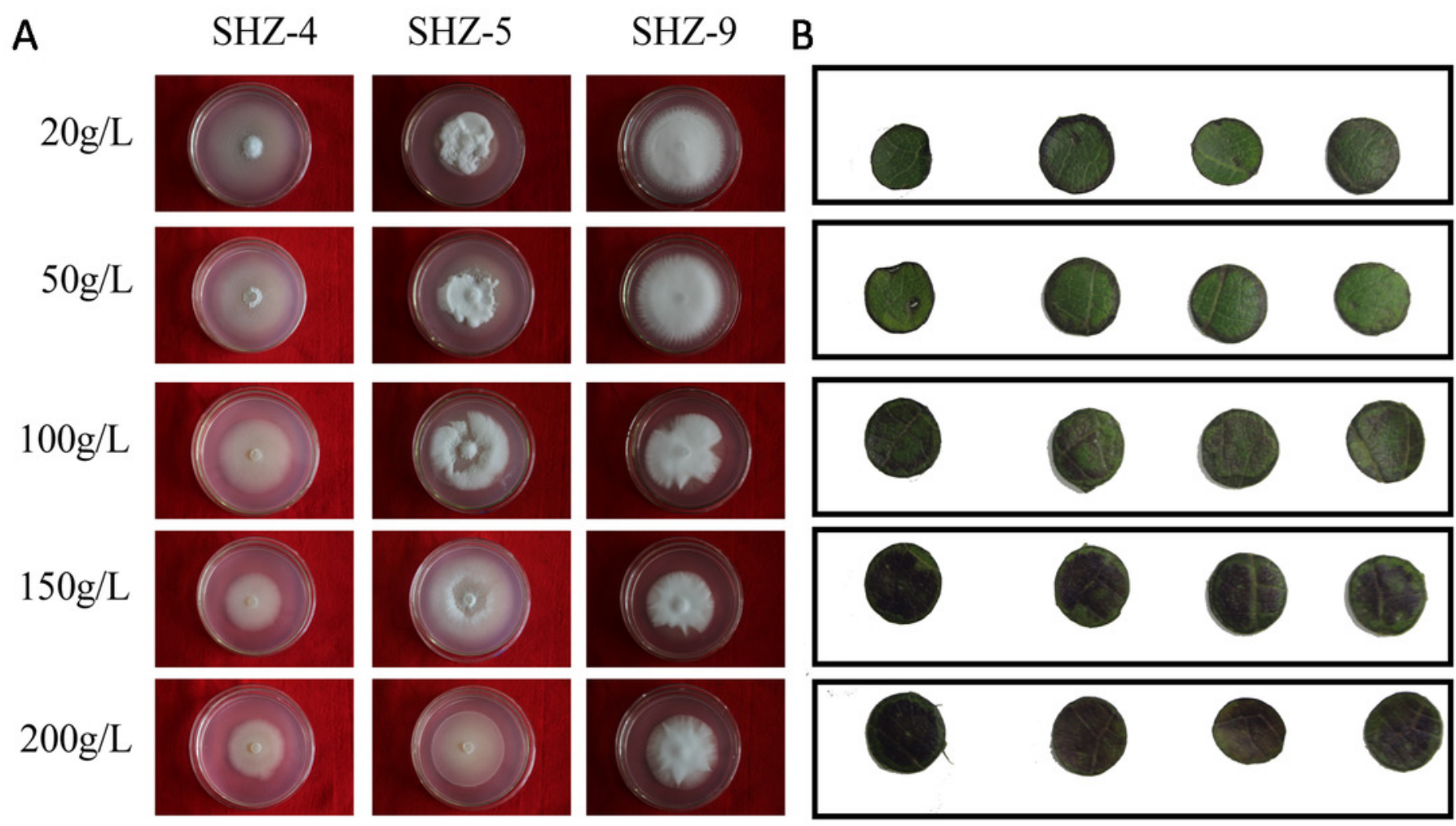




\section{Table 1 (on next page)}

egetative compatibility test of Nit mutants

Note: the " + "represent the interstrain pairings with testers successed to yield prototrophic growth at the line of mycelial contact. 
Table 1. Vegetative compatibility test of Nit mutants

\begin{tabular}{cccccccc}
\hline & \multicolumn{7}{c}{ NitM } \\
\cline { 2 - 6 } Nit1 & SHZ- & SHZ- & SHZ- & SHZ- & SHZ- & SHZ- & VCGs \\
& 2 & 4 & 11 & 8 & 5 & 9 & \\
\hline SHZ-2 & + & - & + & - & - & - & VCG2 \\
\hline SHZ-13 & - & - & - & - & - & - & VCG3 \\
\hline SHZ-4 & - & + & - & - & + & $+/-$ & VCG1 \\
\hline SNZ-18 & + & - & + & - & - & - & VCG2 \\
\hline SHZ-5 & - & - & - & + & + & - & VCG1 \\
\hline SHZ-6 & - & + & - & + & $+/-$ & - & VCG1 \\
\hline SHZ-21 & - & - & - & - & - & - & VCG3 \\
\hline SHZ-8 & - & + & - & + & + & + & VCG1 \\
\hline SHZ-9 & - & $+/-$ & - & - & + & + & VCG1 \\
\hline SHZ-11 & - & - & + & - & - & + & VCG1 \\
\hline
\end{tabular}

Note: the " + "represent the interstrain pairings with testers successed to yield prototrophic growth at the line of mycelial contact. 
Table 2 (on next page)

Pathogenicity differentiation test on cotton plants infected with $V$. dahliae 
1

2 Table 2. Pathogenicity differentiation test on cotton plants infected with $V$. dahliae

\begin{tabular}{cccccc}
\hline V & \multicolumn{3}{c}{ Disease index } & Average \\
\cline { 2 - 4 } dahliae & Xinluzao 7 & Zhongmian 35 & Xinluzao 33 & value \\
\hline SHZ-9 & 78.06 & 48.89 & 26.67 & 51.20 \\
SHZ-16 & 77.22 & 46.94 & 28.33 & 50.83 \\
SHZ-13 & 69.44 & 50.00 & 30.33 & 49.93 \\
SHZ-7 & 75.00 & 42.22 & 30.33 & 49.19 \\
SHZ-21 & 69.44 & 45.56 & 25.33 & 46.78 \\
SHZ-3 & 72.22 & 35.00 & 23.33 & 43.52 \\
SHZ-20 & 48.06 & 33.70 & 24.67 & 35.48 \\
SHZ-18 & 50.56 & 35.28 & 19.67 & 35.17 \\
SHZ-6 & 50.83 & 34.44 & 19.33 & 34.87 \\
SHZ-22 & 50.83 & 32.59 & 21.00 & 34.81 \\
SHZ-11 & 44.72 & 38.06 & 21.33 & 34.70 \\
SHZ-5 & 53.61 & 31.11 & 15.33 & 33.35 \\
SHZ-8 & 41.67 & 34.44 & 21.33 & 32.48 \\
SHZ-15 & 48.89 & 28.89 & 19.33 & 32.37 \\
SHZ-23 & 46.67 & 29.63 & 18.33 & 31.54 \\
SHZ-1 & 56.11 & 23.33 & 14.33 & 31.26 \\
SHZ-2 & 43.61 & 21.11 & 14.00 & 26.24 \\
SHZ-19 & 23.70 & 30.00 & 11.00 & 21.57 \\
SHZ-10 & 25.56 & 28.33 & 10.33 & 21.41 \\
SHZ-24 & 20.74 & 28.33 & 11.33 & 20.14 \\
SHZ-4 & 22.22 & 27.22 & 9.67 & 19.70 \\
SHZ-14 & 21.85 & 23.89 & 9.33 & 18.36 \\
\hline & & & & \\
\hline
\end{tabular}

3

4 